\title{
Substrate binding activates the designed triple mutant of the colicin E7 metallonuclease
}

Eszter Németh $^{[\mathrm{a}, \mathrm{b}]}$, Tamás Körtvélyesi ${ }^{[\mathrm{b}]}$, Milan Kožíšek ${ }^{[\mathrm{c}]}$, Peter W. Thulstrup ${ }^{[\mathrm{d}]}$, Hans E.M.

Christensen $^{[\mathrm{e}]}$, Masamitsu Asaka ${ }^{[\mathrm{f}]}$, Kyosuke Nagata ${ }^{[\mathrm{f}]}$, and Béla Gyurcsik*[a,g]

aDepartment of Inorganic and Analytical Chemistry, University of Szeged, Dóm tér 7, H-6720 Szeged, Hungary

${ }^{b}$ Department of Physical Chemistry and Material Sciences, University of Szeged, Aradi Vértanúk tere 1, H-6720 Szeged, Hungary

${ }^{\mathrm{c}}$ Institute of Organic Chemistry and Biochemistry, Academy of Sciences of the Czech Republic, Gilead Sciences and IOCB Research Center, Flemingovo namestí 2, 16610 Prague 6, Czech Republic

${ }^{\mathrm{d} D e p a r t m e n t}$ of Chemistry, University of Copenhagen, Universitetsparken 5, DK 2100 Copenhagen, Denmark

eDepartment of Chemistry, Technical University of Denmark, Kemitorvet, Building 207, 2800 Kgs. Lyngby, Denmark

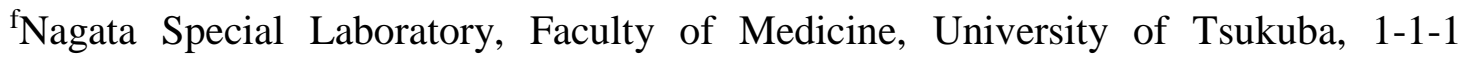
Tennodai, Tsukuba 305-8575, Japan

'MTA-SZTE Bioinorganic Chemistry Research Group, Dóm tér 7, H-6720 Szeged, Hungary

*Corresponding authors' e-mail: gyurcsik@chem.u-szeged.hu, phone number: +36$62-544335$ 


\begin{abstract}
The nuclease domain of colicin E7 (NColE7) cleaves DNA nonspecifically. The active centre is a $\mathrm{Zn}^{2+}$-containing $\mathrm{HNH}$ motif at the C-terminus. The $\mathrm{N}$-terminal loop is essential for the catalytic activity providing opportunity for allosteric modulation of the enzyme. To identify the key residues responsible for the structural integrity of NColE7, a virtual alanine scan was performed on a semi-empirical quantum chemical level within the 25 residue long N-terminal sequence (446-470). Based on the calculations the T454A/K458A/W464ANColE7 triple mutant (TKW) was expressed and purified. According to the agarose gel electrophoresis experiments and linear dichroism spectra the catalytic activity of the TKW mutant decreased in comparison with wild type NColE7. The distorted structure and weakened $\mathrm{Zn}^{2+}$-binding may account for this as revealed by circular dichroism spectra, mass spectrometry, fluorescence based thermal analysis and isothermal microcalorimetric titrations. Remarkably, the substrate induced the folding of the mutant protein.
\end{abstract}




\section{Introduction}

Colicin E7 is a toxin released by Escherichia coli cells for protection against related bacteria $[1,2]$. With the aid of the receptor binding and membrane translocation domains, the nuclease domain (NColE7) enters the target cell and digests DNA nonspecifically [2]. The host cell protects itself by co-expressing the $\operatorname{Im} 7$ immunity protein, which blocks the DNA binding site of the nuclease $[3,4]$. The catalytic centre of the nuclease is a $\mathrm{Zn}^{2+}$-containing $\mathrm{HNH}$ motif [5-7] at the C-terminus. The metal ion required for the hydrolytic activity is coordinated by the H544, H569 and H573 residues in tetrahedral geometry. The fourth coordination site of the $\mathrm{Zn}^{2+}$-ion is occupied by a water molecule or by the substrate DNA [8]. The attacking nucleophile is an $\mathrm{OH}^{-}$-ion generated from a water molecule by the side-chain of H545 [9], which is one of the most conserved residues in the HNH protein family. The protonation of the leaving group is still a matter of discussion [10].

The HNH-motif is a well conserved $\beta \beta \alpha$ metal binding unit [6]. This kind of structure is common in biology, e.g. in the transcription factor zinc finger (ZF) proteins. In ZFs the $\mathrm{Zn}^{2+}$-ion has a mixed Cys/His coordination in tetrahedral geometry, and instead of being catalytically active it induces a protein structure suitable for DNA-binding [11,12]. In contrast to this $\mathrm{NColE7}$ has a preorganized $\mathrm{Zn}^{2+}$-binding site as suggested by the results of $\mathrm{CD}$ spectroscopic studies showing only minor changes upon the removal of $\mathrm{Zn}^{2+}$-ion by EDTA $[13,14]$. The crystal structures of apo NColE7/DNA complexes $[15,16]$ are also similar to the NColE7 structures containing $\mathrm{Zn}^{2+}$-ion [3,4,9,17-19].

The presence of the $\mathrm{N}$-terminal loop is essential for the catalytic activity its role being multiple. Point mutations within this region affecting the positively charged residues (K446, R447 or K449) resulted in low activity enzyme variants [14,20]. Previously we showed that the N-terminally shortened $\Delta \mathrm{N} 4-\mathrm{NColE7}$ is inactive due to the lack of the 446-KRNK-449 sequence [13,21]. However, the solution structure, the $\mathrm{Zn}^{2+}$ and the DNA binding was not significantly influenced by the exchange or deletion of the 446-KRNK-449 residues. At the same time, the deletion of 25 residues at the $\mathrm{N}$-terminus of the protein decreased both the $\mathrm{Zn}^{2+}$ and DNA binding affinity [13]. This points to the extensive interactions between the $\mathrm{N}$ terminal sequence and the $\mathrm{HNH}$-motif resembling to an allosteric regulation. Such property could be exploited to design a controlled artificial nuclease for the purpose of DNA modifications [22].

The main scope of the present work was to identify residues of high impact on the catalytic behavior within the 450-470 N-terminal sequence of NColE7. Based on the results of 
semiempirical quantum chemical computations the TKW protein was designed with T454A/K458A/W464A triple mutation. The structure, function, $\mathrm{Zn}^{2+}$ and $\mathrm{DNA}$ binding properties of the mutant protein were investigated by various techniques including electrophoretic mobility shift, CD and LD spectroscopy, ITC and thermal shift assay.

\section{Materials and Methods}

\section{Computational methods}

Chain $A$ from the crystal structure $1 \mathrm{M} 08$ of the $\mathrm{Zn}^{2+}$-bound NColE7 [3] was applied as the starting geometry for NColE7. Alanine mutations were built in the optimized NColE7 structure manually and the resulted models were optimized as well. All proteins had uncapped termini (i.e. $\mathrm{NH}_{3}{ }^{+}$and $\mathrm{COO}^{-}$groups). Semiempirical quantum chemical computations were performed with the PM6 method [23,24] implemented in MOPAC2009 [25]. Localized molecular orbitals were applied by the MOZYME [26] method. The solvation was considered by COSMO method [27] with the dielectric constant of 78.4. The geometry optimization was carried out by the L-BFGS method after the initial minimization of the hydrogen positions. The gradient norm was $1.0 \mathrm{kcal} / \mathrm{mol} / \AA$. The thermodynamic parameters were computed with the PM6 method, and recalculated in one SCF cycle with the correction PM6-DH2 [28,29].

\section{Cloning, protein expression and purification}

The gene of the triple mutant protein (the sequence shown later in Fig. 3.) was amplified by PCR from the pQE70 plasmid (a generous gift of Prof. K.-F. Chak, Institute of Biochemistry and Molecular Biology, National Yang Ming University, Taipei, Taiwan - [3]) containing the gene of NColE7 and the $\operatorname{Im} 7$ protein. The PCR fragment was cloned into a pGEX-6P-1 vector that provides an N-terminal GST fusion. The plasmid containing the target gene was transformed into E. coli $\mathrm{DH} 10 \mathrm{~B}$ cells and E. coli BL21 (DE3) cells for cloning and protein expression, respectively. The procedure described earlier [14] was applied for protein purification. All the proteins were stored in $20 \mathrm{mM}$ HEPES, at $\mathrm{pH}=7.7$.

\section{Monitoring of DNA binding and cleavage by gel electrophoresis}

In the protein-DNA binding studies the concentration of the 13 base pair oligonucleotide was $0.2 \mu \mathrm{M}$, and the protein final concentration ranged between 0 and $7 \mu \mathrm{M}$. The ${ }^{31}$ P-radiolabelled DNA was hybridized from one single oligonucleotide with complementary sequence at the ends forming a loop upon self hybridization. The samples 
were run on $6 \%$ native polyacrylamide gel electrophoresis (PAGE) at $4{ }^{\circ} \mathrm{C}$. The reaction mixture contained $4 \mathrm{mM} \mathrm{NaCl}$ and $4 \mathrm{mM}$ HEPES buffer $(\mathrm{pH}=7.9)$. The DNA cleavage reaction was inhibited by $100 \mu \mathrm{M}$ EDTA chelating the metal ion that is essential for catalytic activity [8].

The cleavage of $74 \mu \mathrm{M}$ (base pairs) pUC19 plasmid by proteins $(2.8 \mu \mathrm{M})$ in the presence of $2.8 \mu \mathrm{M}$ zinc(II)-acetate was followed by agarose gel electrophoresis. Incubation time before electrophoretic run was $0-60 \mathrm{~min}$ at $37{ }^{\circ} \mathrm{C}$. The CT-DNA cleavage assay contained DNA $(68 \mu \mathrm{M}$, base pairs), protein $(2.6 \mu \mathrm{M})$ and zinc(II)-acetate $(2.6 \mu \mathrm{M})$. Incubation time was varied between 0 and $180 \mathrm{~min}$ at $37^{\circ} \mathrm{C} .10 \mu \mathrm{l}$ aliquots were loaded onto an ethidium bromide containing $1 \%$ agarose gel with $2 \mu$ of $6 \times$ DNA Loading Dye (Thermo Sci.). The electrophoresis was performed in a Bio-Rad Wide Mini Sub Cell ${ }^{\circledR}$ GT system at 6.7 $\mathrm{V} / \mathrm{cm}$ in TAE buffer (40 mM Tris, $20 \mathrm{mM}$ acetic acid, and $1 \mathrm{mM}$ EDTA, $\mathrm{pH}=8.0$ ). For comparison $6 \mu 1$ of the Bio-Rad $1 \mathrm{kbp}$ marker DNA was also loaded to the gel.

\section{Mass spectrometry}

Mass spectrometric (MS) measurements were performed on a LCT Premier (Waters) instrument equipped with a nanoflow electrospray ionization (nano-ESI) source and a time-offlight (TOF) analyzer in positive ion mode as described earlier [13][14]. The recorded $\mathrm{m} / \mathrm{z}$ data were deconvoluted using the MassLynx ${ }^{\mathrm{TM}} \mathrm{v} 4.1$ (Waters) software equipped with the MaxEnt1 algorithm. The high charge states of the multiply charged spectrum, ranging from +10 to +17 , were used to calculate the apparent mass.

\section{Isothermal microcalorimetric titrations}

Isothermal calorimetric titrations were performed with the MicroCal Auto-iTC 200 (GE Healthcare Life Sciences) instrument. The protein samples $(\sim 50 \mu \mathrm{M})$ were dialyzed for 12 hours in 7000 MWCO Thermo Scientific Slide-A-Lyzer casettes against $20 \mathrm{mM}$ cacodylate buffer, $\mathrm{pH}=7.0$. The concentration of the protein after dialysis was determined by HPLC amino acid analysis. $\mathrm{ZnCl}_{2}$ was dissolved in the same buffer $(\mathrm{c}=400 \mu \mathrm{M})$. The dilution heat of $\mathrm{ZnCl}_{2}$ with the buffer was determined for each experiment and the integrated data of dilution heats were subtracted from the corresponding data of protein titrations. $200 \mu 1$ of protein solutions were titrated with $2 \mu \mathrm{l}$ aliquots of $400 \mu \mathrm{M} \mathrm{ZnCl}_{2}$ up to $40 \mu \mathrm{l}$ and a spacing of $240 \mathrm{~s}$. Prior to titrations the plates were centrifuged to avoid bubbles in the samples. 
Thermal stability of the proteins was measured in a ROCHE LightCycler 480 instrument. The Sypro Orange (Invitrogen) fluorescent dye was applied in $625 \times$ dilution to detect protein unfolding. The protein concentration was $0.4 \mathrm{mg} / \mathrm{ml}$ with a twofold molar excess of $\mathrm{ZnCl}_{2}$, in $20 \mathrm{mM}$ cacodylate buffer, $\mathrm{pH}=$ 7.0. Samples were heated from 20 to 95 ${ }^{\circ} \mathrm{C}$, with $4.40{ }^{\circ} \mathrm{C} / \mathrm{s}$.

\section{Linear dichroism spectroscopy}

Flow linear dichroism spectra were measured on a Jasco-815 CD spectrometer equipped for linear dichroism spectroscopy (LD) as described in [30]. CT-DNA cleavage was followed in reaction mixtures containing $130 \mu \mathrm{M}$ (base pairs) CT-DNA and $5 \mu \mathrm{M}$ TKW with 5,25 or $50 \mu \mathrm{M}$ zinc(II)-acetate in a microvolume Couette flow device. The samples contained $17.7 \mathrm{mM} \mathrm{KH} \mathrm{KHO}_{4}$ and $2.5 \mathrm{mM}$ HEPES buffer, $\mathrm{pH}=7.7 .70 \mu \mathrm{L}$ aliquots of the sample incubated at $37{ }^{\circ} \mathrm{C}$ were used to record LD spectra at room temperature. Control experiments were conducted with CT-DNA in the presence or absence of zinc(II)-acetate, TKW, EDTA or Im7. Protein binding was studied with a $130 \mu \mathrm{M}$ (final concentration calculated for base pairs) CT-DNA sample. The reaction mixture contained $60 \mu \mathrm{M}$ EDTA to inhibit nuclease digestion, $17 \mathrm{mM} \mathrm{KH} \mathrm{PO}_{4}, 2.4 \mathrm{mM}$ HEPES (the $\mathrm{pH}$ was adjusted to 7.7 ) and $0-5 \mu \mathrm{M}$ protein. The solutions were incubated for $10 \mathrm{~min}$ before recording the spectra. Longer incubation times did not influence the results.

\section{SRCD spectroscopy}

The Synchrotron Radiation Circular Dichroism (SRCD) spectra were recorded at the CD1 beamline of the storage ring ASTRID at the Institute for Storage Ring Facilities (ISA), University of Aarhus, Denmark [31,32]. Camphor-sulfonic acid served as a calibration material for the instrument. All spectra were recorded with $1 \mathrm{~nm}$ steps and a dwell time of $2 \mathrm{~s}$ per step, using a $100.4 \mu \mathrm{m}$ quartz cell (SUPRASIL, Hellma GmbH, Germany), in the wavelength range of $175-260 \mathrm{~nm}$. The concentration of the protein solutions was adjusted to $3.2 \times 10^{-5} \mathrm{M}$ in $10 \mathrm{mM}$ HEPES, $\mathrm{pH}=7.7$. From raw spectra the water baseline was subtracted.

\section{Results}

Design of mutations by computational alanine scan 
Virtual alanine scan was carried out within the 25 residues long $\mathrm{N}$-terminal sequence of NColE7 - i.e. residues 446-470 - to obtain an estimate of the contribution of each residue to the structural stability of NColE7. The effect of the exchange of each amino acids - except for Gly and Ala - to Ala was monitored one at a time. The alignment of the optimized structures of alanine mutants to that of the native protein resulted in small differences (RMSD for the whole sequences aligned in PyMol was between 0.022 and $0.174 \mathrm{~nm}$ ). The highest deviation from the optimized NColE7 was observed for R447A. This is in agreement with the low activity of R447 mutants demonstrated previously [14][20].

Next the $\Phi$ backbone dihedral angle between the $25^{\text {th }}$ and $26^{\text {th }}$ residues $(470 \mathrm{~K}$ and $471 \mathrm{D}$, respectively) was altered from $290^{\circ}$ to $30^{\circ}$ so, that the $\mathrm{N}$-terminal sequence turned away from the core of the protein (Fig. 1a). The geometry of the distorted structure was optimized. The contribution of the N-terminal loop to protein stability was estimated as the difference in the enthalpy of formation of the distorted $\left(\Delta H_{f, \text { distorted }}\right)$ and folded $\left(\Delta H_{f, f o l d e d}\right)$ forms for each alanine mutant, as given by equation (1).

$$
\Delta \Delta H_{f}=\Delta H_{f, \text { distorted }}-\Delta H_{f, \text { folded }}
$$

The value computed in this way is independent from the number of atoms in the molecule, reflecting the interactions of the N-terminal segment with the core protein or solvent. The comparison of the results obtained for each alanine mutant allowed an estimate of the contribution of each residue of the $\mathrm{N}$-terminal sequence (Fig. 1b).

\section{Fig. 1.}

The expected $\Delta \Delta H_{f}$ is positive, because the folded protein is more stable than its distorted counterpart. The opposite was found in calculations with PM6 without the DH2 complement (see Table S1. in ESI) demonstrating the importance of the DH2 correction in calculations of proteins. The N-terminal 446-KRNK-449 sequence was examined previously [14] and therefore, was not considered here. The lowest $\Delta \Delta H_{f}$ was found for W464A mutant. This tryptophan contributes to the stability of N-terminal chain by hydrophobic interactions and forms hydrogen bond with the carbonyl oxygen of P475. K458A interacts with V476 while T454 with Y556 or D557. The latter interaction between the $\mathrm{N}$-terminal sequence and the HNH-motif (Fig. 2.) may affect the structure of the catalytic site and thereby the nuclease 
activity. Consequently, the triple alanine mutant T454A/K458A/W464A (TKW) was selected for experimental study.

\section{Fig. 2.}

\section{Expression and purification of the TKW mutant}

The TKW mutant and the wild type NColE7 were N-terminally fused to a GST protein to allow for affinity based purification on a glutathione sepharose column. 8 residues remained at the N-terminus of the proteins (Fig. 3) after the cleavage of GST tag, having no effect on the catalytic activity [14].

\section{Fig. 3.}

To avoid the cytotoxic effect, the proteins were coexpressed with the $\operatorname{Im} 7$ immunity protein, which was separated at $\mathrm{pH}=3.0$ by cation exchange chromatography. The protein was obtained in its apo form as a consequence of the protonation of the histidine residues at low $\mathrm{pH}$. The observed molecular mass was 15610.9 Da for TKW (the calculated value for the apo form is $15610 \mathrm{Da}$ ).

\section{Nuclease activity}

The nuclease activity of the TKW mutant in comparison with NColE7 was studied by agarose gel electrophoresis (Fig. 4). While in the presence of NColE7 the supercoiled form of the pUC19 plasmid disappeared before loading the sample to the gel (lane 0 min in Fig. 4a), TKW could only cleave a fraction of the supercoiled plasmid within one hour. Fig. $4 \mathrm{~b}$ shows the cleavage of CT-DNA sample containing only linear DNA by the TKW mutant.

\section{Fig. 4.}

The reaction was also followed by flow linear dichroism (FLD) spectroscopy, a technique that is suitable to study fiber like-molecules (Fig. S1). The experiments confirmed that the catalytic activity of TKW is lower than that of NColE7. The activity was similar to those of the proteins containing N-terminal point mutations within the 446-KRNK-449 sequence, and was in the order of KGNK > TKW > KGNG . The protein folding, $\mathrm{Zn}^{2+}$ and DNA binding affinity was not affected by the $\mathrm{N}$-terminal mutations suggesting the direct role 
of the N-terminal positively charged residues in the catalytic mechanism [14]. However, these amino acids are not affected in the TKW mutant, so its low nuclease activity may be attributed to other reasons: 1) improper protein folding 2) decreased metal binding affinity 3) disturbed substrate binding and 4) modification of functional residues. The investigation of these aspects is presented in the following sections.

\section{Protein conformation}

SRCD spectra of NColE7 and TKW recorded under the same conditions reflected that the mutations drastically affect the $\mathrm{CD}$ pattern (Fig. 5). The fractions of the secondary structure elements estimated from the SRCD spectrum for NColE7 are as follows: $31 \%$ and $12 \%$ for $\alpha$-helices and $\beta$-sheets, respectively [33]. This is in good agreement with the values obtained form the crystal structure of NColE7 (PDB ID 1M08): 38\% of $\alpha$-helices and 16\% of $\beta$-sheets [34]. At the same time the significantly different SRCD spectral pattern of TKW mutant yields $13 \% \alpha$-helices and $21 \% \beta$-sheets. As the $\mathrm{N}$-terminal sequence forms a loop its mutation is not expected to cause such a large deviation from the native protein. This suggests that the interactions of the T454, K458 and W464 residues are widespread and their mutation affected the overall protein structure. Similarly, CD spectral changes were reported with a W140A mutant of the staphylococcal nuclease [35] emphasizing the importance of the Trp residues in protein folding [36].

The stability of the TKW and NColE7 protein structures was compared in a thermal shift assay using the Sypro Orange fluorescent dye (Invitrogen). The fluorescence of the dye is quenched by water, but once it binds to the hydrophobic regions of the unfolded protein, the fluorescence is increased. The melting point of NColE7 was $59^{\circ} \mathrm{C}$ (Fig. S2) being the same as earlier published for colicin E9 [37]. The decrease of the fluorescence intensity in the thermal curve of TKW supported that the protein is already partially unfolded resulting in uncertain melting point determination.

\section{$\mathrm{Zn}^{2+}$-binding}

SRCD spectra of TKW in the presence of increasing $\mathrm{Zn}^{2+}$ concentration showed that not even ten equivalents of the metal ion could saturate the protein. Nevertheless, the CD pattern approached that of the NColE7- $\mathrm{Zn}^{2+}$ complex. Upon further addition of $\mathrm{Zn}^{2+}$-ions the intensity of the spectra decreased and presumably partial denaturation of the protein occurred. 
This demonstrated deleterious influence of the mutations on the protein structure including the active centre with significantly decreased metal ion affinity.

\section{Fig. 5.}

The differences in $\mathrm{Zn}^{2+}$-binding were also well reflected in the ESI-MS spectra. The observed molecular mass for NColE7 (15874.9) corresponded to 100 \% holo form (expected mass: 15877.2) after the addition of one equivalent $\mathrm{Zn}^{2+}$-ion [14]. However, the mass spectra of TKW with one equivalent of $\mathrm{Zn}^{2+}$ showed a dual distribution with only around one third of the protein in the holo form. Based on $\mathrm{m} / \mathrm{z}$ spectra, the structure of the apo protein is probably less stable than the holo form, and thus highly charged after the ionization process. (Fig. S3).

Isothermal microcalorimetric titration experiments were performed to quantitatively describe the $\mathrm{Zn}^{2+}$ binding of the proteins (Fig. 6). The data obtained from curve fitting are shown in Table 1. Titrations were performed only in cacodylate buffer, as the low solubility of $\mathrm{Zn}^{2+}$-ions at neutral $\mathrm{pH}$ limited the buffer selection. Therefore, we could not determine buffer independent enthalpy, proton linkage during complex formation and the entropy contribution. The curve fitting procedure for NColE7 and the reason for omitting the measurement points below the $\mathrm{Zn}^{2+}:$ NColE7 $\sim 0.6$ molar ratio was discussed in detail in our previous paper [14]. Both proteins bound $\mathrm{Zn}^{2+}$ with 1:1 stoichiometry. The $K_{\mathrm{d}}$ values are 16.6 $\pm 3.5 \mathrm{nM}[14]$ and $7.7 \pm 0.4 \mu \mathrm{M}$ for NColE7 and TKW, respectively. The $\mathrm{Zn}^{2+}$ binding ability of the mutant protein decreased by three orders of magnitude in agreement with SRCD and mass spectrometric findings. The enthalpic change was also lower for the mutant protein, than for NColE7.

\section{Fig. 6.}

\section{Table 1.}

The reason for the weak $\mathrm{Zn}^{2+}$-binding probably lies in the structural changes observed by SRCD-spectroscopy that influence the structure of the active site of the protein. The preorganized metal binding site in NColE7 is unstable in TKW and can only partially be rescued by the addition of $\mathrm{Zn}^{2+}$-ions. Consequently, the $\mathrm{N}$-terminal loop must have a structural role supporting the proper folding of the C-terminal HNH motif. 
The binding of the DNA substrate was investigated by native PAGE gel mobility shift assay of a 13 base pairs long PCR fragment. Similar concentrations of the native and the mutant protein were necessary to shift the DNA band (Fig. 7). The calculated $K_{\mathrm{d}}$ vales related to the DNA/protein complexes were $0.16 \mu \mathrm{M}$ for NColE7 [14] and $0.14 \pm 0.05 \mu \mathrm{M}$ for TKW. This indicated that surprisingly, there was no difference in the strength of DNA binding.

\section{Fig. 7.}

The DNA binding was further studied by flow linear dichroism (FLD) spectroscopy, with the large size CT-DNA substrate. The FLD signal is sensitive to protein binding: the conformational changes (e.g. bending of the DNA and the overall shape of the complex) alter the orientation ability. The FLD intensities measured at $260 \mathrm{~nm}$ was plotted against the protein concentration in Fig. 8. The binding of both proteins resulted in disappearance of the LD signal. For TKW a slightly higher protein:DNA ratio was needed in accordance with the gel mobility shift results. This again implies that the DNA-binding affinity of TKW is maintained, even though it is not properly folded as revealed above by its SRCD spectrum (see Fig. 5). Titration of CT-DNA by protein in the presence of the Hoechst fluorescent dye showed that TKW replaces the dye at higher concentration than NColE7 (Fig. S4.)

\section{Fig. 8.}

Considering the low activity and widespread changes in the conformation of the TKW mutant, it is surprising that only minor difference was found in DNA-affinity. A possible explanation was obtained by SRCD spectroscopy. The spectrum of the NColE7/DNA complex was identical to the sum of the component spectra. Thus, no significant conformational change was detected in agreement with the identical crystal structures of the protein in the presence and absence of DNA [15-17]. Although the folding of TKW has significantly been affected by the mutations, the SRCD spectrum of the TKW/DNA complex showed very similar pattern to that of the NColE7/DNA complex. This suggested that an NColE7-like structure of the TKW mutant has been induced by the interaction with DNA. Similar induced folding of a truncated staphylococcal nuclease has been observed upon addition of its inhibitor to the unfolded protein [38].

The effect of the addition of one equivalent $\mathrm{Zn}^{2+}$-ions to the protein/DNA complexes on the SRCD spectral pattern was comparable for both the NColE7 and TKW proteins (Fig. 
9) showing that the DNA-binding mediated the preformed structure of the HNH motif, and thereby contributed to the stronger $\mathrm{Zn}^{2+}$-binding.

\section{Fig. 9.}

\section{Conclusions}

The contribution of the N-terminal loop to the stabilization of NColE7 structure and catalytic function was studied through the TKW triple mutant protein (T454A/K458A/W464A). The residues for mutation study were selected considering the results of semi-empirical quantum chemical calculations and available crystal structures. The chosen residues were expected to be responsible for interactions between the N-terminal region and the core of the protein. The catalytic activity of TKW significantly decreased, similarly to the N-terminal point mutants affecting the positive charged residues in the 446KRNK-449 sequence [14]. ITC measurements revealed that the TKW mutant had approximately $2000 \times$ weaker $\mathrm{Zn}^{2+}$-binding than $\mathrm{NColE7}$ being also reflected in $\mathrm{MS}$ and SRCD results. A decreased $\mathrm{Zn}^{2+}$ binding with $K_{\mathrm{d}}$ in $\mu \mathrm{M}$ range was also observed previously for the inactive $\triangle \mathrm{N} 25-\mathrm{NColE7}$ mutant [13], in which the N-terminal sequence - including also the T454, K458 and W464 residues - was deleted. A partially unfolded structure of TKW was demonstrated by SRCD, MS and fluorescence based stability measurements. The conformational changes most probably also affected the structure of the C-terminal $\mathrm{HNH}$ motif causing the weak $\mathrm{Zn}^{2+}$ binding. SRCD spectra also pointed out that the conformation of TKW approaches that of the wild type enzyme upon $\mathrm{Zn}^{2+}$-addition, but the protein can not be fully loaded by the metal ion. Surprisingly, the DNA binding affinity was only slightly affected by the mutations. Furthermore, the NColE7-like structure was induced by DNAbinding. All the above results indicated that the presence of the T454, K458 and W464 residues within the $\mathrm{N}$-terminal loop in NColE7 significantly contribute to the proper function of the protein. Even though these amino acids do not belong to the DNA binding part or to the active centre, they contribute to the proper folding and stabilize the structure of the metalbinding HNH-motif. This supports that NColE7 is a promising candidate for the design of a new controlled artificial nuclease. As shown in this work, a careful design of point mutations permits the intramolecular allosteric modulation of the enzymatic activity. The DNAmediated folding of TKW leading to the preorganization of the metal binding site is an additional advantageous feature that could be exploited in the redesign of NColE7. 


\section{Acknowledgements}

This work has received support through the Hungarian Science Foundation (OTKANKTH CK80850), European Union and the State of Hungary, co-financed by the European Social Fund in the framework of TÁMOP 4.2.4.A/2-11-1-2012-0001 'National Excellence Program', TÁMOP-4.2.2.C-11/1/KONV-2012-010, TÁMOP-4.2.2.A-11/1/KONV-20120047. The computational resources at HPC of University Szeged and financial support from the CALIPSO Programme (FP7/2007-2013, grant $\mathrm{n}^{\mathrm{o}}$ 312284) is also acknowledged. B.G. and E.N. thanks JSPS. M.K. was partially funded by EU OPPC program CZ.2.16/3.1.00/24016. 


\section{References}

[1] Chak K, Kuo W, Lu F, James R (1991) J Gen Microbiol 137:91-100.

[2] Lin Y, Liao C, Liang P, Yuan H, Chak K (2004) Biochem Biophys Res Commun 318:8187.

[3] Cheng Y, Hsia K, Doudeva LG, Chak K, Yuan HS (2002) J Mol Biol 324:227-236.

[4] Sui M, Tsai L, Hsia K, Doudeva L, Ku W, Han G, Yuan H (2002) Protein Sci 11:29472957.

[5] Orlowski J, Bujnicki JM (2008) Nucleic Acids Res 36:3552-3569.

[6] Eastberg JH, Eklund J, Monnat R, Jr., Stoddard BL (2007) Biochemistry 46:7215-7225.

[7] Mehta P, Katta K, Krishnaswamy S (2004) Protein Sci 13:295-300.

[8] Ku W, Liu Y, Hsu Y, Liao C, Liang P, Yuan H, Chak K (2002) Nucleic Acids Res 30:1670-1678.

[9] Doudeva L, Huang D, Hsia K, Shi Z, Li C, Shen Y, Cheng Y, Yuan H (2006) Protein Sci 15:269-280.

[10] Hsia K, Li C, Yuan H (2005) Curr Opin Struct Biol 15:126-134.

[11] Blasie CA, Berg JM (2002) Biochemistry 41:15068-15073.

[12] Li W, Zhang J, Wang J, Wang W (2008) J Am Chem Soc 130:892-900.

[13] Czene A, Németh E, Zóka IG, Jakab-Simon NI, Körtvélyesi T, Nagata K, Christensen HEM, Gyurcsik B (2013) J Biol Inorg Chem 18:309-321.

[14] Németh E, Körtvélyesi T, Thulstrup PW, Christensen HEM, Kožíšek M, Nagata K, Czene A, Gyurcsik B (2014) Protein Sci 23:1113-1122.

[15] Hsia K, Chak K, Liang P, Cheng Y, Ku W, Yuan HS (2004) Structure 12:205-214.

[16] Wang Y-, Wright J, D., Doudeva LGH-, Lim C, Yuan HS (2009) J Am Chem Soc 131:17345-17353.

[17] Wang Y, Yang W, Li C, Doudeva LG, Yuan HS (2007) Nucleic Acids Res 35:584-594.

[18] Huang H, Yuan HS (2007) J Mol Biol 368:812-821.

[19] Ko T, Liao C, Ku W, Chak K, Yuan HS (1999) Structure 7:91-102.

[20] Shi Z, Chak K, Yuan H (2005) J Biol Chem 280:24663-24668. 
[21] Czene A, Tóth E, Gyurcsik B, Otten H, Poulsen JN, Lo Leggio L, Larsen S, Christensen HEM, Nagata K (2013) Acta Crystallogr Sect F Struct Biol Cryst Commun 69:551-554.

[22] Gyurcsik B, Czene A (2011) Future Med Chem 3:1935-1966.

[23] Stewart JJP (2009) J Mol Model 15:765-805.

[24] Stewart JJP (2007) J Mol Model 13:1173-1213.

[25] Stewart JJP (2008) MOPAC2009, Colorado Springs, Stewart Computational Chemistry, http://openmopac.net.

[26] Stewart JJP (1996) Int J Quantum Chem 58:133-146.

[27] Klamt A, Schüürmann G (1993) J Chem Soc Perkin Trans 2:799-805.

[28] Korth M, Pitonak M, Rezac J, Hobza P (2010) J Chem Theory Comput 6:344-352.

[29] Rezac J, Fanfrlik J, Salahub D, Hobza P (2009) J Chem Theory Comput 5:1749-1760.

[30] Marrington R, Dafforn T, Halsall D, Rodger A (2004) Biophys J 87:2002-2012.

[31] Miles AJ, Hoffmann SV, Tao Y, Janes RW, Wallace BA (2007) J Spectroscopy 21:245255.

[32] Miles AJ, Janes RW, Brown A, Clarke DT, Sutherland JC, Tao Y, Wallace BA, Hoffmann SV (2008) J Synchrotron Radiat 15:420-422.

[33] Sreerama N, Woody RW (2000) Anal Biochem 287:252-260.

[34] Kabsch W, Sander C (1983) Biopolymers 22:2577-2637.

[35] Hu HY, Wu MC, Fang HJ, Forrest MD, Hu CK, Tsong TY, Chen HM (2010) Biophys Chem 151:170-177.

[36] Samanta U, Pal D, Chakrabarti P (2000) Proteins 38:288-300.

[37] Mosbahi K, Walker D, Lea E, Moore G, James R, Kleanthous C (2004) J Biol Chem 279:22145-22151.

[38] Flanagan J, M., Kataoka M, Shortle D, Engelman D, M. (1992) Proc Natl Acad Sci U S A 89:748-752. 
Table 1. Binding parameters ( $\mathrm{n}$ is the average number of the bound metal ions and $\mathrm{K}$ is the apparent stability constant) obtained in the fitting procedure of ITC curves applying one $\mathrm{Zn}^{2+}$ site model. The indicated errors refer to fitting errors.

\begin{tabular}{lll}
\hline $\begin{array}{l}\text { Binding } \\
\text { parameters }\end{array}$ & NColE7 & TKW \\
\hline $\mathrm{n}$ & $1.19 \pm 0.01$ & $1.24 \pm 0.01$ \\
$\mathrm{~K}\left(\mathrm{M}^{-1}\right)$ & $(6.03 \pm 1.23) \times 10^{7}$ & $(1.30 \pm 0.7) \times 10^{5}$ \\
$\Delta \mathrm{H}(\mathrm{kcal} / \mathrm{mol})$ & $-32.9 \pm 0.2$ & $-22.7 \pm 0.3$ \\
$\Delta \mathrm{S}(\mathrm{cal} / \mathrm{mol} / \mathrm{K})$ & -74.7 & -52.8 \\
\hline
\end{tabular}


Figure legends

Fig. 1. a) Overall structure of the original (brown) and partially unfolded (blue) of a mutant NColE7 protein. b) Contribution of the $\mathrm{N}$-terminal sequence to protein stability was estimated by equation (1) at PM6 level with DH2 correction for all mutants.

Fig. 2. The location and interactions of the amino acids selected for the experimental mutation study. T454, K458, W464 and the interacting counterparts are shown by sticks in the X-Ray structure with PDB Id: 1M08 [3]. Distances between selected atoms are shown in $\AA$.

Fig. 3. Sequence of the purified proteins - NColE7 was expressed for comparison. The remaining part of the GST-tag after the protease cleavage is in blue. The N-terminal sequence studied in the virtual alanine scan is underlined. The mutations are indicated by red and the $\mathrm{HNH}$ motif by green color. The $\mathrm{Zn}^{2+}$-binding histidines are highlighted by yellow, while the general base in the catalytic reaction - H545 - by blue background.

Fig. 4. a) The cleavage of $74 \mu \mathrm{M}$ (base pairs) pUC19 plasmid by $2.8 \mu \mathrm{M}$ proteins in the presence of $2.8 \mu \mathrm{M}$ zinc(II)-acetate. The substrate was incubated for $0-60$ or $0-180 \mathrm{~min}$ at 37 ${ }^{\circ} \mathrm{C}$ with NColE7 or TKW, respectively prior to loading onto the gel. b) Cleavage of $68 \mu \mathrm{M}$ (base pairs) CT-DNA by $2.6 \mu \mathrm{M}$ TKW and $2.6 \mu \mathrm{M}$ zinc(II)-acetate at $37{ }^{\circ} \mathrm{C}$. The $1 \mathrm{~kb}$ molecular ruler (BioRad) was used as a reference on both gels.

Fig. 5. SRCD spectra of the NColE7 (green) and TKW mutant (light blue) proteins $(31,6 \mu \mathrm{M}$ in $20 \mathrm{mM}$ HEPES, $\mathrm{pH}$ 7.4) and the titration of the proteins with $\mathrm{Zn}^{2+}$-ions (the latter curves were corrected for dilution). The arrows show the effect of increasing protein: $\mathrm{Zn}^{2+}$ ratio from $1: 0$ to $1: 10$.

Fig. 6. Isothermal microcalorimetric titrations of $50 \mu \mathrm{M}$ NColE7 (a) and TKW (b) with $\mathrm{Zn}^{2+}-$ ions, in $20 \mathrm{mM}$ cacodylate buffer, $\mathrm{pH}=7.0$.

Fig. 7. Substrate binding of NColE7 and [14] TKW proteins to a 13 bp hybridized oligonucleotide followed by gel mobility shift assay. The nuclease activity was inhibited by the addition of $0.5 \mathrm{mM}$ EDTA. 
Fig. 8. Changes in CT-DNA (130 $\mu \mathrm{M}$ / base pairs) FLD signal intensity at $260 \mathrm{~nm}$ as a consequence of protein-DNA interaction. The spectra were recorded after short incubation of ca. $5 \mathrm{~min}$ at room temperature in the presence of $0.5 \mathrm{mM}$ EDTA. Absorbance measurements (data not shown) confirmed that the decrease of FLD signal is not related to the loss of DNA by precipitation.

Fig. 9. The SRCD spectra of NColE7 (orange) and TKW (31,6 $\mu \mathrm{M}$ in 20mM HEPES, pH = 7.4) in the presence of DNA (light blue in the absence of $\mathrm{Zn}^{2+}$-ions and dark blue with one equivalent of $\mathrm{Zn}^{2+}$-ions). All curves were corrected for dilution. 
Fig. 1.

a)

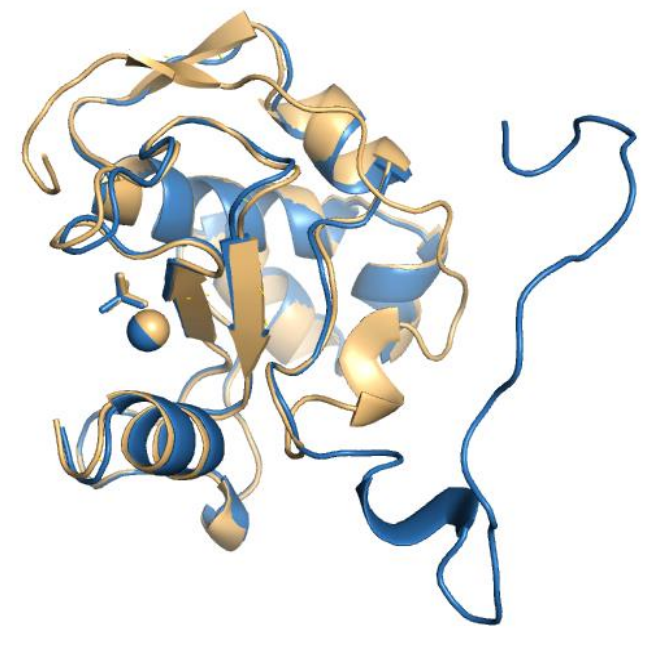

b)

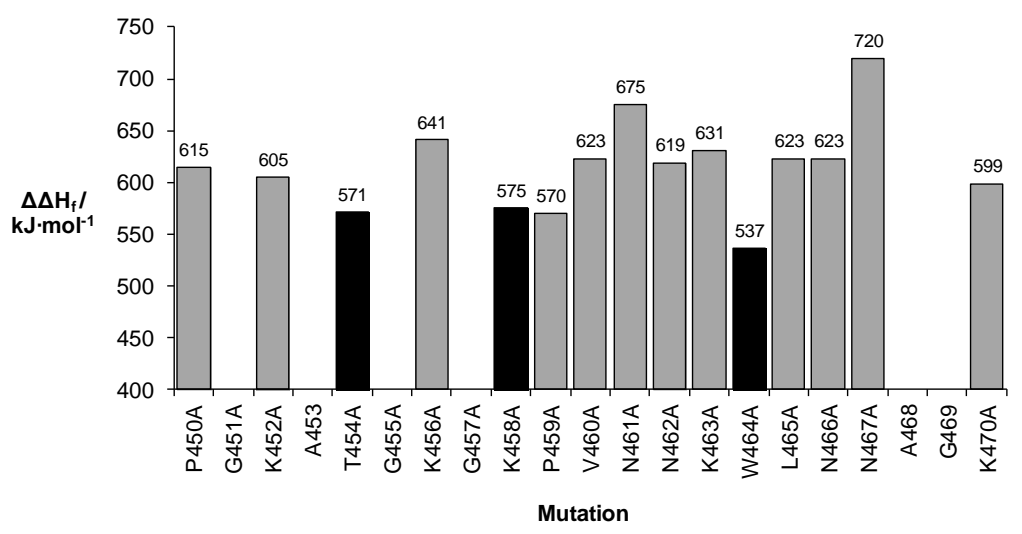


Fig. 2.

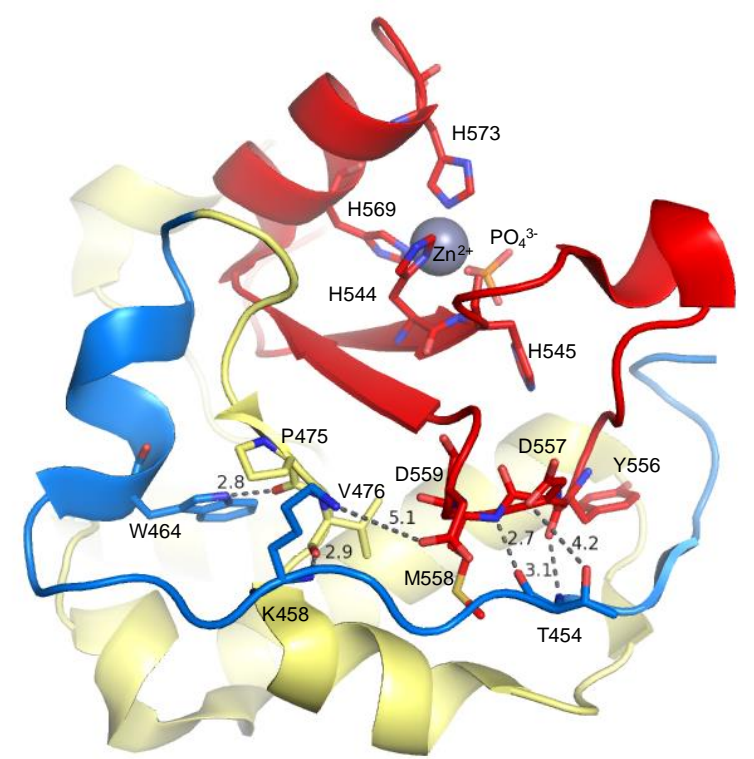


Fig. 3.

NCOIE7 438-GPLGSPEFKRNKPGKATGKGKPVNNKWLNNAGKDLGSPVPDRIANKLRDKEFKSFDDFRKKFWEEVSKDPEL TKW 438-GPLGSPEFKRNKPGKAAGKGAPVNNKALNNAGKDLGSPVPDRIANKLRDKEFKSFDDFRKKFWEEVSKDPEL

NCOIE7 SKQFSRNNNDRMKVGKAPKTRTQDVSGKRTSFELHHEKPISQNGGVYDMDNISVVTPKRHIDIHRGK-576 TKW SKQFSRNNNDRMKVGKAPKTRTQDVSGKRTSEELHHEKPISQNGGVYDMDNISVVTPKRHIDIHRGK-576 
Fig. 4.

a)

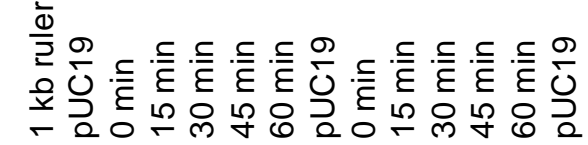

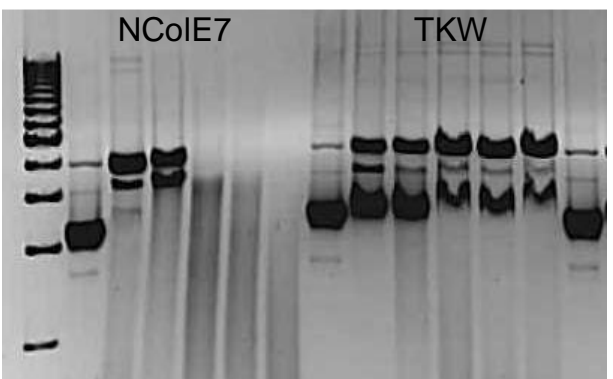

b)

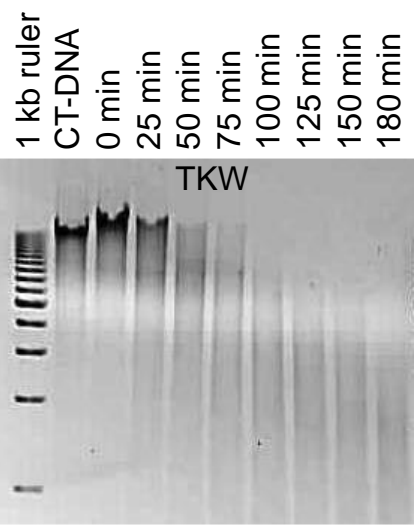


Fig. 5.

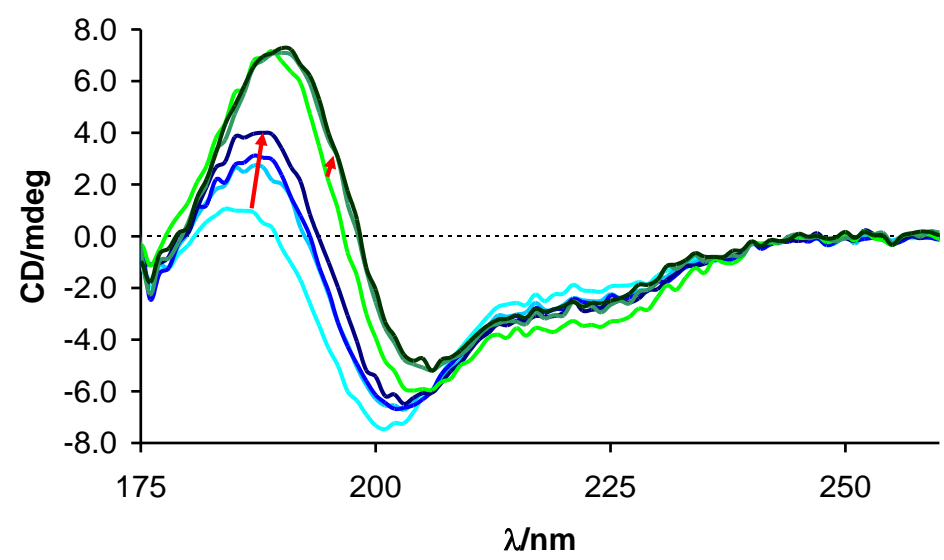


Fig. 6.

a)

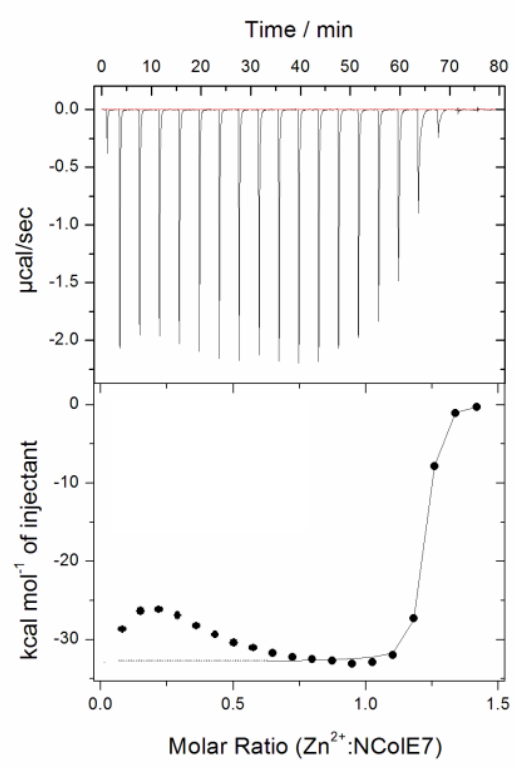

b)

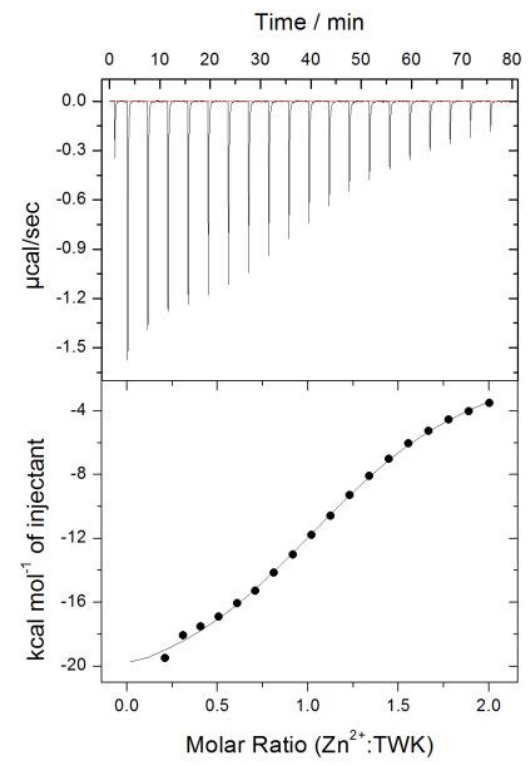


Fig. 7.

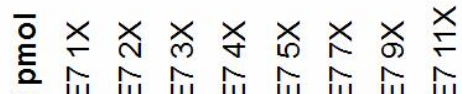

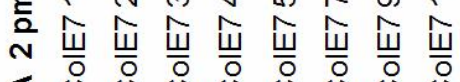

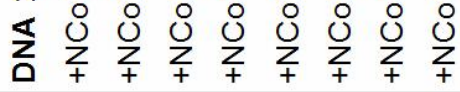

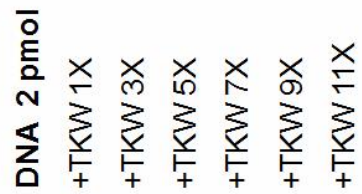

APmpA

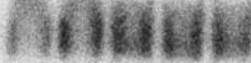


Fig. 8.

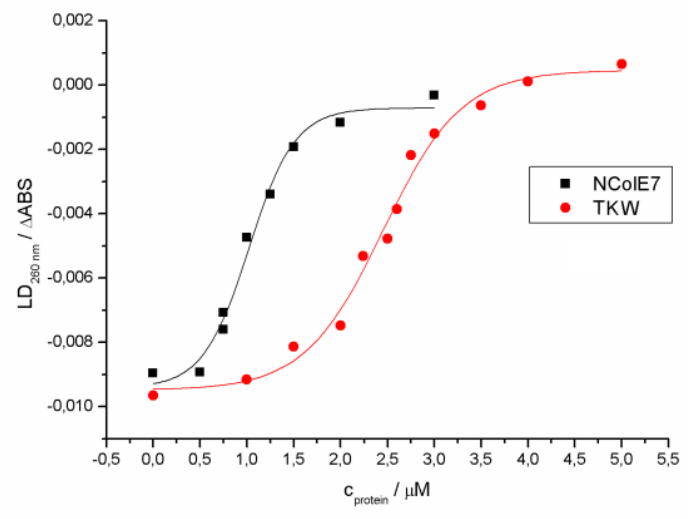


Fig. 9.

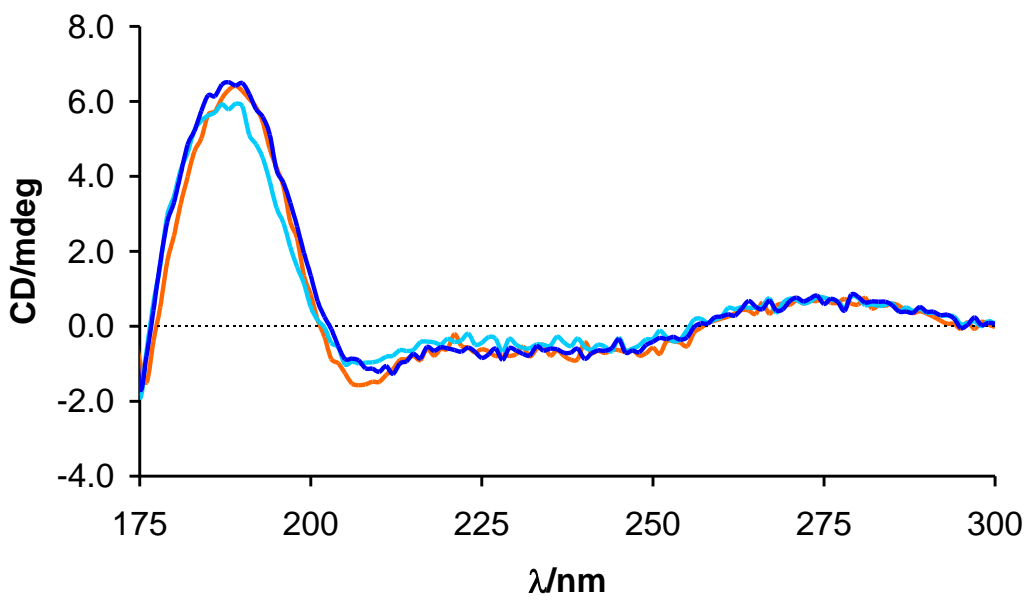

